\title{
Linx
}

Revue des linguistes de l'université Paris X Nanterre

$75 \mid 2017$

Imaginaires de la ponctuation. Ordre et inquiétude du discours

\section{Imaginaire de la ponctuation dans l'écriture contemporaine. Une enquête}

\section{Stéphane Bikialo}

\section{(2) OpenEdition}

\section{Journals}

Édition électronique

URL : http://journals.openedition.org/linx/1900

DOI : $10.4000 /$ linx.1900

ISSN : 2118-9692

Éditeur

Presses universitaires de Paris Nanterre

Édition imprimée

Date de publication : 22 décembre 2017

Pagination : 107-126

ISBN : 978-2-84016-308-4

ISSN : 0246-8743

Référence électronique

Stéphane Bikialo, «Imaginaire de la ponctuation dans l'écriture contemporaine. Une enquête », Linx [En ligne], 75 | 2017, mis en ligne le 23 novembre 2018, consulté le 30 avril 2019. URL : http:// journals.openedition.org/linx/1900 ; DOI : 10.4000/linx.1900

Ce document a été généré automatiquement le 30 avril 2019.

Département de Sciences du langage, Université Paris Ouest 


\title{
Imaginaire de la ponctuation dans l'écriture contemporaine. Une enquête
}

\author{
Stéphane Bikialo
}

1 En 1980, dans un numéro de Langue française consacré à la ponctuation, Annette Lorenceau proposait le compte-rendu et l'analyse d'une enquête adressée à «quatrevingt écrivains dont les livres étaient exposés dans une grande librairie parisienne » (1980: 88) : quarante avaient répondu, dont vingt de manière un peu développée, au questionnaire suivant :

1. Pensez-vous qu'il existe actuellement des règles générales de ponctuation?

2. Ont-elles, selon vous, plus de rapports avec l'oral ou avec la syntaxe?

3. Consultez-vous un manuel ? Lequel ?

4. Avez-vous des habitudes de ponctuation qui vous soient particulières?

5. Mettez-vous la ponctuation dès le premier jet ? La corrigez-vous beaucoup ?

6. Vous arrive-t-il de lire à haute voix pour mettre la ponctuation?

7. Corrigez-vous la ponctuation sur les épreuves?

8. Les éditeurs et imprimeurs respectent-ils la ponctuation de vos manuscrits?

9. Vous attachez-vous à la faire respecter? Quelles sont vos remarques sur ce point?

2 L'enjeu, en 1980, était notamment de faire reconnaitre la ponctuation comme objet d'étude linguistique - ce qui est acquis désormais, mais tardivement comme le souligne Anis (2004). Par ailleurs, et le vocabulaire employé dans les questions en témoigne, le présupposé est qu'il existe une opposition entre ce qui relève des « règles » (« syntaxe », « manuel », « corrigez », « respecter ») et de «l'oral ». Lorenceau achève son article par une dichotomie entre les quelques (rares) auteur.trice.s qui ne voient dans la ponctuation qu'une norme grammaticale à suivre, avant de citer toutes les formes de considérations esthétiques, concluant par :

Nous voilà bien loin de la grammaire, de la syntaxe, des règles. Respiration, rythme, cadence, temps, ton, mélodie, musique, souffle, serpentement, ruissellement, mouvements, gestes, intonations, silence, style, voilà les mots qui reviennent sous la plume des écrivains. Plus proches de la tradition orale du XVIII ${ }^{\mathrm{e}}$ siècle que des 
contraintes grammaticales que voulaient imposer les imprimeurs du XIX ${ }^{\mathrm{e}}$, les écrivains $\mathrm{du} \mathrm{XX}^{\mathrm{e}}$ siècle nous ouvrent des perspectives très nouvelles - et inattendues - sur la ponctuation, phénomène qui reste mineur pour les grammairiens et les linguistes. La richesse très grande de cette enquête, la remise en cause qu'elle révèle, la recherche d'un outil nouveau dont on ressent un besoin évident, doivent nous engager à poursuivre les recherches dans ce domaine, à demander aux grammairiens d'intégrer dans leurs travaux cette ponctuation dont Beauzée, grammairien-philosophe du XVIII ${ }^{e}$ siècle, disait qu'elle était « la partie essentielle de l'orthographe » $(1980: 97)$.

D'objet d'étude émergent, la ponctuation est devenue un enjeu linguistique et littéraire essentiel : formes discursives non verbales mais verbalisables, écrites mais étroitement liées à l'oral, lieux d'un imaginaire normatif très fort et en même temps au cœur des recherches formelles les plus novatrices, les signes de ponctuation sont centraux dans l'attention portées aux matérialités discursives, chez les linguistes comme chez les auteurs ou autrices. Les trente dernières années ont vu en effet une attention renouvelée ou diversifiée à cette matérialité - sans doute en partie encouragée, voire contrainte par le développement du numérique, le fait que l'écran provoque de nouvelles pratiques d'écritures ou (les deux n'étant pas exclusifs) qu'il incite à une attention accrue à la page papier. C'est pourquoi des aspects comme le rapport à l'oral (questions 2 et 6) et aux normes grammaticales et/ou typographiques, éditoriales (questions 1, 3, 7, 8, 9) m'ont semblé devoir être réinterrogées, notamment en partant de l'hypothèse d'un travail sur l'espace graphique, la « part visuelle du dire» (Meschonnic, $1982: 304$ ) et sa dimension rythmique.

4 Julien Rault et moi avons ainsi actualisé l'enquête, en établissant un questionnaire orienté par ce qui nous a semblé représenter des enjeux linguistiques contemporains de la ponctuation, et plus précisément d'un imaginaire de la ponctuation chez ces scripteur.se.s de métier que sont les auteurs et autrices d'œuvres littéraires. Il s'agissait, en réunissant autour des mêmes questions un ensemble de personnes ayant en commun d'avoir écrit au moins un livre publié, de voir si émergeaient des représentations communes de la ponctuation au sein d'une pratique littéraire au début du XXI ${ }^{e}$ siècle. Le questionnaire était le suivant :

1 : A quel(s) mot(s) associez-vous spontanément ou immédiatement celui de ponctuation?

2 : La ponctuation a-t-elle pour vous et dans votre écriture une dimension plutôt sonore/orale ou graphique/visuelle?

3 : La ponctuation est-elle d'abord liée à «l'ordre du discours" (règles, normes, contraintes syntaxiques et discursives) ou à «l'inquiétude du discours " (Foucault)?

4 : Quel est pour vous le lien entre le rythme (intérieur et/ou de l'écriture) et la ponctuation?

La synthèse qui suit porte sur une quarantaine de réponses, obtenues d'auteurs et d'autrices qui ont tous et toutes en commun de pratiquer une littérature de création, qui s'opposerait à une littérature de commande, ce qui se caractérise par une attention aux formes et aux représentations ${ }^{1}$ : Eric Arlix, Andréas Becker, François Bégaudeau, Marcel Bénabou, Pierre Bergounioux, François Bon, Jean-Paul Chabrier, Patricia Cottrond'Aubigné, François Cusset, Christophe Dabitch, Didier Daeninckx, Michel Deguy, Louise Desbrusses, Kossi Efoui, Antoine Emaz, Annie Ernaux, Isabelle Garron, Jean-Pierre Girard, Alain Glykos, Jacques Jouet, Leslie Kaplan, Koffi Kwahulé, David Léon, Daniel Lewin Becker, Gérard Macé, Jean-Charles Massera, Laurent Mauvignier, Xabi Molia, Bernard Noël, Adeline Picault, Jean-Luc Raharimanana, Philippe Rahmy, Laurine Rousselet, Lydie 
Salvayre, Tiphaine Samoyault, Joachim Séné, Jean-Pierre Siméon, Martine Sonnet, Catherine Ternaux, Joël Vernet. A ce corpus de réponses directes à l'enquête, j'ai adjoint quelques réflexions importantes d'auteurs et d'autrices sur la ponctuation (Claude Simon, Louise Desbrusses, Maylis de Kérangal...) mentionnées au sein de travaux sur la ponctuation, ce qui est une manière, outre d'élargir le corpus, de situer ce compte-rendu d'enquête par rapport aux nombreux travaux récents sur le sujet.

Un (ou des) imaginaire(s) contemporain(s) spécifique(s) aux usages littéraires de la ponctuation émerge-t-il ? Dans quelle mesure cet imaginaire de la ponctuation est-il relié - explicitement ou non - à un imaginaire de la langue, et des normes qui en actualisent certains traits? Plus peut-être que d'autres signes linguistiques, la ponctuation donne lieu à des imaginaires, des représentations véhiculées par les textes, les œuvres et sédimentées par l'histoire. Elle est un espace de la langue privilégié d'expression des discours épilinguistiques, qu'on peut définir avec Canut (2007: 50-51) comme des « discours autonomes sur les formes langagières ", sur les langues ou les pratiques. C'est à partir de ces commentaires épilinguistiques sollicités - voire orientés, par les questions que je vais synthétiser quelques traits de l'imaginaire contemporain de la ponctuation dans le discours littéraire.

\section{Synthèse de l'enquête}

\section{1. «À quel(s) mot(s) associez-vous spontanément ou immédiatement celui de ponctuation? »}

7 À cette première question très ouverte, les réponses sont de deux types : a) les auteurs répondent avec des notions génériques (en particulier respiration, souffle, rythme) ; b) ils s'attachent à tel ou tel signe, soit en évoquant un signe qui prend le dessus sur les autres et semble une sorte de désignateur prototypique, archétypal de la ponctuation: en particulier le point (Picault, Sonnet) et la virgule (Mauvignier, Arlix), soit en pensant les signes en opposition, orientant vers leur statut de ponctème ou de signe plérémique ${ }^{2}$ :

(1) Je pourrais seulement dire que les : et les; ne font pas partie de mon bestiaire.

J'ai une affection toute intime pour les virgules qui me font pourtant tellement de problèmes. J'hésite toujours lorsque je dois en "mettre une ", comme j'hésite à poser une pièce sur un jeu d'échec. (Glykos)

(2)! mort

. arrêt

; hésitation

, pause

/ froid

() renforcement

? inutile, tout est question

- fluidité

« inutile, tout est entre parenthèses (Becker)

(3) Le système classique des pauses, autrement dit l'ensemble , ;: !?

On peut y ajouter le tiret (que j'aime bien pour son élasticité), le / (que je n'aime pas trop mais il peut se révéler pratique), les ( ) (que je n'aime pas, elles trouent le texte sans pour autant être silencieuses). (Emaz)

(4) Chacun nos petits usages. Personnellement j'ai usé pendant un temps du pointvirgule un peu à la façon du double-point de Montaigne, et puis je l'ai supprimé radicalement. Je déteste les points d'exclamations, je les trouve vulgaire. J'aime 
bien que la question soit la matière même de l'écriture et donc me dispense de

l'usage du ? (Bon) les traits /+ animés / en (3) et (4) (parenthèses silencieuses, point d'exclamation vulgaire) soulignent que la ponctuation est l'espace d'expression d'un imaginaire très fertile, d'une part, et d'un investissement affectif, voire intime, d'autre part : affection toute intime (1); que j'aime bien ; que je n'aime pas (3); je déteste (4). Apparait nettement par ailleurs que la ponctuation est pensée comme signe, avec son signifiant et son signifié (polysémique) au sein d'un système de signes, la valeur de chaque signe étant définie par opposition à d'autres signes (de ponctuation ou verbaux). Les réponses à cette première question soulignent ainsi l'importance de l'ancrage en langue des signes de ponctuation (Molia répond d'ailleurs à cette question par un mot : « langue »), encore trop peu abordé dans les études linguistiques. Elles soulignent également le lien très fort entre ponctuation et rythmes (sonore et visuel).

\subsection{La ponctuation a-t-elle pour vous et dans votre écriture une dimension plutôt sonore/orale ou graphique/visuelle?}

Les réponses sont très équilibrées, la dimension sonore étant dominante pour neuf des auteurs et autrices interrogé.e. $s^{3}$, la visuelle pour huit autres, en particulier Sonnet (« Graphique et visuelle, participant à la mesure, à l'équilibre et à l'élégance du texte tant que l'écriture reste sur le papier ou sur l'écran.»), et Bon, qui insiste sur la structure en blocs typographiques :

(5) M'intéresse beaucoup plus le saut de paragraphe, Gertrude Stein en parle dans le toujours pas traduit How to write. Très important pour moi les œuvres monobloc (Thomas Bernhard, ou Broch de La mort de Virgile) mais ce n'est pas une remarque accessoire : récemment Kobo et iTunes nous refusaient un texte d'Arnaud Maïsetti parce qu'ils considéraient que si un texte dépassait 40 pages sans paragraphe, il y avait bug.

Il m'arrive souvent d'écrire en bloc sans paragraphe, c'est même pour moi une notion de pavé qui me concerne esthétiquement, mais quand le texte se développe je découpe en $\S$ disjoints, et c'est ce qui me permet d'en reprendre la rhétorique et les attaques.

La conception prosodique longtemps dominante ${ }^{4}$ semble s'atténuer par la prise en compte de la dimension graphique. Cette prise en compte du signifiant graphique n'a rien d'une évidence, comme a pu le montrer Testenoire (2016) sur les résistances à l'égard de la «langue écrite» chez Saussure et Bally, la parution encore très novatrice et minoritaire en 1983 des travaux d'Anis (1983) sur le signifiant graphique, ou encore la bipartition, ou l'hésitation, entre "ponctuation » et "typographie " présente dans la plupart des travaux évoquant la dimension visuelle ${ }^{5}$, en particulier ceux de Catach, qui distingue la mise en page (" ensemble de techniques visuelles d'organisation et de présentation de l'objet-livre, qui vont du blanc des mots aux blancs des pages, en passant par tous procédés intérieurs et extérieurs au texte») de la ponctuation (" ensemble des signes visuels d'organisation et de présentation accompagnant le texte écrit, intérieurs au texte et communs au manuscrit et à l'imprimé » $(1980: 21)$. Il faudra attendre les travaux de Védénina (1989) et de Favriaud (2000) pour que la ponctuation blanche soit définitivement incluse dans la ponctuation. 
11 Au niveau de la création, si les poètes ont fait du blanc un enjeu - presque un marqueur poétique depuis Mallarmé et Reverdy, les auteur.e.s en prose soit limitent (pour des raisons économiques ${ }^{6}$ ) leurs audaces graphiques, soit dissocient encore mise en page - du côté des typographes, de "l'énonciation éditoriale " (Arabyan, 2016) - et signes de ponctuation. Si dans l'enquête de Lorenceau, plusieurs évoquent le blanc, l'enquête présente a généralisé cette attention, une grande majorité des réponses évoquant les deux dimensions (sonore et visuelle) : "Les deux, indissociablement » (Salvayre); «Les deux absolument » (Mauvignier) ; " Difficile de séparer les deux » (Cusset) ; " On ne peut pas séparer les deux dimensions, tout est sonore et graphique, oral et visuel » (Becker) :

(6) Dans mon langage, hormis la perception imaginaire, logique, visuelle, la perception sonore/orale est prépondérante. J'ai toujours écrit en récitant. Je ne sépare pas l'oralité de l'écrit. (Rousselet)

Dans ces cas, la tendance est plutôt d'assimiler la ponctuation noire au sonore et la ponctuation blanche au visuel :

(7) La ponctuation noire a une fonction uniquement sonore pour moi. [...] la blanche fonctionne sur les deux plans, plutôt dans son aspect sonore (Cottron d'Aubigné)

(8) Sonore/orale pour ce qui est des virgules, tirets, points, puisqu'ils interviennent rythmiquement. Et graphique/visuelle avec les sauts de ligne, les blancs, coupures

[...] comme effet de ruptures, de relances, de déplacements, etc. (Mauvignier)

Dans cette double réponse ou dans l'impossibilité de réponse globale apparaissent souvent des commentaires soulignant la nécessité de penser selon les signes (9), les œuvres (10) ou les genres (18) :

(9) Difficile à dire. Pour la virgule, je dirais «sonore » (rythmique). Pour d'autres aspects, comme la mise en forme des dialogues, c'est plutôt une affaire visuelle. (Bégaudeau)

(10) Tout dépend du texte. Parfois, elle tente d'opérer dans une dimension exclusivement orale (United Emmerdements of New Order, We Are L'Europe), parfois visuelle (Jean de La Ciotat - La légende). (Massera)

\subsection{La ponctuation est-elle d'abord liée à «l'ordre du discours » (règles, normes, contraintes syntaxiques et discursives) ou à «l'inquiétude du discours » (Foucault)?}

14 Même si « les écrivains ne font pas de grammaire, ils font la langue, et c'est bien assez ${ }^{7}$, la grammaire configure chez la plupart des auteurs et autrices, comme chez tout.e scripteur.se, un imaginaire de la langue, spontanément normatif en ce qui concerne la ponctuation. Ainsi, dans les réponses recueillies, treize mentionnent l'ordre, vingt-et-un l'inquiétude, et sept ne savent pas ou évoquent les deux.

15 Les écrivain.e.s de ce début du XXI ${ }^{e}$ siècle sont les héritiers du " moment grammatical » (1890-1940) de la littérature mis au jour par Gilles Philippe dans Sujet, verbe, complément ; celui-ci décrit ainsi comment, de Maupassant à Barthes, en passant par Claudel, Gide, Aragon, Sartre, etc., s'est opéré un «changement d'épistémè littéraire de toute une génération » $(2002: 31)$ où les auteurs et autrices utilisent des notions syntaxiques pour penser, et parler de, la littérature ; ce moment grammatical est à mettre en lien avec la grammaticalisation de la ponctuation, entamée au XVIII ${ }^{\mathrm{e}}$ siècle mais qui s'étend surtout aux XIX et $\mathrm{XX}^{\mathrm{e}}$ siècles avec le passage d'une conception phonocentriste à une conception autonomiste (Rault, 2014b). Au confluent de cette grammaticalisation à la fois de la 
manière d'envisager l'écriture et des signes de ponctuation s'affirme un imaginaire très normatif de la ponctuation chez les auteurs et autrices :

(11) Le rapport à la ponctuation est un rapport à une norme, et pose, très concrètement, des questions engageant un certain positionnement par rapport à cette norme. Notamment lors de la phase de correction (dite aussi « préparation ») d'un livre en cours de fabrication. Je dois ajouter que mon usage de la ponctuation, notamment dans les textes théoriques (articles, essais), est plutôt du côté de la mise en ordre, car il vise la clarté du propos, organise la hiérarchie des différentes étapes de raisonnement. (Bégaudeau)

(12) Si j'emploie en prose le système normal des pauses, c'est bien un discours «normal» que je vise. La ponctuation devient comme invisible, elle est où on l'attend et fait son travail de marqueur syntaxique, de clarification. Si je passe à prose avec tirets seuls, prose non ponctuée ou vers libre, c'est autre chose, sans doute un signal de poésie, en tout cas d'une autre saisie du langage avec une fonction qui n'est pas celle de la communication quotidienne. (Emaz)

(13) Je ne souhaite pas la subvertir mais l'utiliser telle qu'elle existe, avec les possibilités qu'elle nous offre. [...] La ponctuation m'apparaît comme le soulignement, le marquage, de ce travail sur la syntaxe, la possibilité d'en rendre immédiatement visibles certains aspects. (Pireyre)

Apparait la prégnance d'un imaginaire et d'une intériorisation des emplois normatifs de la ponctuation mais aussi le sentiment que la ponctuation est un espace formel privilégié pour rendre visible, ou prendre ses distances avec, ces normes, notamment en fonction des genres : essai, prose (romanesque) et poésie (en prose ou vers). La ponctuation, en raison de sa dimension originellement normative (liée à la clarté, à l'organisation du discours), est précisément un enjeu pour faire passer de l'ordre à l'inquiétude du discours, de l'organisation logique d'une pensée " posée ", " arrêtée » qui " organise et fixe la pensée » (Siméon en (22)) à une manière d'inquiéter le sens, d'interroger, d'ouvrir :

(14) Elle relève plutôt d'un ordre normatif intériorisé, mais ces règles étant impossibles à fixer (relatives à un état de la langue, à un style, au statut des textes, à un référent dans l'histoire littéraire aussi) elles débouchent sur une normativité fuyante, ou impossible, qui oui, « inquiète » le texte. (Cusset)

(15) C'est un jeu perpétuel entre ces deux pôles. Respect de la norme avec l'emploi du tiret en retrait pour signifier la prise de parole, ou majuscule d'attaque de phrase par exemple, mais utilisation sauvage de la majuscule pour sonner l'alerte. (Daeninckx)

17 C'est au sein de l'œuvre, par glissement (14) ou contraste (15), qu'une sorte de système de la ponctuation se met en place, comprenant à la fois la dimension normative et la dimension esthétique de la ponctuation ${ }^{8}$, que le questionnaire place du côté de l'inquiétude du discours. Ce terme est employé par M. Foucault au début de L'Ordre du discours : cette inquiétude est liée à « ce qu'est le discours dans sa réalité matérielle de chose prononcée ou écrite ${ }^{9}$; la norme est plutôt de l'ordre de ce qui ne se voit pas, dont la matérialité n'est plus sensible voire esquivée. C'est ce qu'exprime L. Desbrusses soulignant le rôle de la matérialité graphique :

(16) si une ponctuation ordinaire semble invisible parce qu'elle répond aux habitudes de lecture, dès qu'il y a rupture par rapport aux usages courants, c'est probablement la dimension graphique qui est perçue la première : l'œil, avant de se poser sur le premier mot, se promène sur la page et en note consciemment ou pas, les aspects inhabituels. (Desbrusses, $2015: 107-108)$

Si le discours littéraire peut se caractériser comme relevant de, ou plutôt générant, une forme d'inquiétude du discours, on comprendra qu'une majorité d'auteurs et d'autrices évoquent la ponctuation comme un des enjeux de cette inquiétude : 
(17) Toujours à l'inquiétude. C'est une sorte d'échafaudage qui tient autant que possible le texte qui flirte constamment avec les abîmes. (Becker)

(18) À l'inquiétude du discours, puisqu'elle défend le souci, l'emphase, la passion, la nécessité, mais surtout l'urgence. La ponctuation procède vraiment de l'urgence du discours et de la parole, de ce qui s'écrit et va se porter en voix (Picault)

On retrouve ici à la fois ce qui relève de l'agencement (17) et de la subjectivité, de la modalisation (18), fonctions majeures de la ponctuation à travers l'histoire comme l'a souligné J. Dürrenmatt (2015: 23-24), qui peuvent être envisagés du côté de l'ordre comme de l'inquiétude du discours. Afin d'approcher cette dimension esthétique, la notion de rythme (qu'il s'agisse de la vitesse comme l'évoque A. Picault avec «l'urgence », ou plus généralement de la subjectivation du discours) apparaît fondamentale.

\subsection{Quel est pour vous le lien entre le rythme (intérieur et/ou de l'écriture) et la ponctuation?}

«La ponctuation n'est qu'un aspect de la question générale, et cruciale, du rythme » écrit Cusset. Une grande partie des réponses à cette question font apparaître, sans surprise, le lien étroit, voire l'indistinction, entre rythme et ponctuation: «Je ne peux pas démêler les choses : tout vient ensemble " (Sonnet). En accord avec la polysémie de la notion de rythme ${ }^{10}$, plusieurs manières d'envisager cette relation sont proposées par les différent.e.s auteurs et autrices :

- Un lien entre rythme et ponctuation autour du corps (respiration, pulsation, battement, souffle) :

(19) C'est un lien viscéral, la ponctuation me permet d'être au plus proche de ce que je veux écrire, d'être fidèle à mon impulsion première et de ne pas amputer le sens. La ponctuation est le battement de ce qui s'écrit, le tempo, c'est un lien très intime qui s'établit entre le rythme intérieur et cette dernière qui fait office de «métronome » et induit une pulsation (Picault)

- Un lien entre rythme et ponctuation autour de la musique : «le son, la musique, trois mots, un rythme », « tripes rythmiques » (Bon) ; « des temps, des noires, des blanches » (Séné) « Il n'y a que le rythme qui compte, la musique d'un texte. La ponctuation donne de la vitesse ou ralentit la lecture, augmente le son ou le baisse » (Becker), « la ponctuation est pour moi le chef d'orchestre » (Becker Levin), «notation musicale, parce que d'une part j'entends le texte en moi. avec son rythme. et sa dynamique et que la ponctuation me permet de le transmettre à la personne qui le lit »(Desbrusses, 2015 : 108). Claude Simon déjà soulignait :

(20) [...] que l'on est souvent amené, uniquement par les nécessités (je dirais même les exigences) musicales de la phrase, à rejeter un mot que l'on croyait juste, ou, au contraire, à rajouter un mot qui, alors, s'avère juste !... Une phrase qui n'est pas "bien balancée" (que le rythme soit long ou court, heurté ou sinueux, peu importe) est, ipso facto, sur le plan du sens, vide, creuse (1977 : 39).

- Un lien entre rythme et ponctuation autour du mouvement, de la relance, de l'élan : «lien majeur, qui souvent relance l'écriture » (Mauvignier) ; «Incise, relance, reprise du souffle » (Macé) ; "Capter le mouvement de la vie» (Daeninckx); "son flux et son reflux» (Salvayre). On retrouve là les éléments posés en 1.2. sur la dimension sonore, orale de la ponctuation.

21 Alain Glykos condense bien ces trois sens principaux, dans sa réponse où interviennent les mots respiration et rythme, termes les plus récurrents au sein des réponses à cette enquête : 
(21) Les signes sont des coups de doigts pour scander la respiration, des coups sur mes doigts pour me contraindre à donner du rythme au texte, des petits métronomes qui contraignent mes élans d'écrire.

\section{Respiration et rythme} adjoint - comme le font certain.e. ${ }^{12}$ - des vocables d'un champ sémantique proche, comme souffle, rythme, ou encore pauss ${ }^{13}$, suspension... Le modèle respiratoire, pneumatique de la respiration semble ainsi inaltérable, des Romains à François Bon. Beauzée, dans l'article « Ponctuation » de l'Encyclopédie (1765) évoque la ponctuation pour les « les besoins de la respiration » et, si cette conception de la ponctuation comme pause respiratoire s'efface peu à peu au XVIII ${ }^{e}$ siècle (Dürrenmatt, 1998: 11), la conception pneumatique ${ }^{14}$ perdure jusqu'au $\mathrm{XX}^{\mathrm{e}}$ siècle, comme en témoigne l'enquête de Lorenceau où la respiration apparaît encore comme une des fonctions essentielles de la ponctuation

[...] le plus grand nombre des écrivains qui ont répondu à l'enquête [...] se réfèrent à une tradition orale de la ponctuation : Claude Roy écrit : «C'est la respiration de la parole qui rythme la ponctuation. J'écris à haute voix. » [...] Gilbert Cesbron : « La ponctuation est aussi indispensable et spontanée que la respiration. Il est vrai que beaucoup de gens ne savent pas respirer » (1980: 94-95).

Les auteurs emploient le mot respiration avec trois orientations principales :

- La respiration comme pause, en accord avec la construction du discours : «Respiration, rythme, suspension, temps, ordre.» (Dabitch) ; « respiration et construction » (Kwahulé) ; « clarté, logique, rythme, modulation » (Chabrier);

- La respiration comme souffle, en lien avec l'investissement du corps dans le discours, autour de cette idée reprise récemment par Maylis de Kayrangal selon laquelle que « la ponctuation est l'anatomie du langage $»^{15}$;

- La respiration comme rythme au sens d'« organisation du mouvement de la parole par un sujet» (Meschonnic, Dessons, 1998: 28), comme chez Noël: «Respiration, Rythme, Orientation, Sens... et si j'osais Sensification!».

Ces trois orientations sont liées; elles reposent sur ce que Meschonnic nomme «physiologisme » en précisant: « De toutes les métaphores du corps, ou de ses fonctions, cette vulgate a privilégié la respiration. La respiration est le cliché majeur du rythme » (1982: 657).

Associer ponctuation et respiration, c'est d'abord créer une analogie entre la langue ou l'œuvre et le corps. Une analogie ancestrale si l'on en croit Louis Marin, qui rappelle, dans De la représentation, que l'imaginaire occidental repose sur un imaginaire du corps omniprésent, ou plutôt un imaginaire de l'absence du corps : tout écrit serait l'indice, le substitut au corps manquant, gardant la trace de ce « corps absent(é) » (Fintz, 2009 : 116).

C'est parce que le langage ne s'enlève pas du corps, et qu'il y a à chercher ce qui en reste dans le discours, que le langage relève non seulement des sciences du langage, de la théorie du langage, mais encore d'une anthropologie. (Meschonnic, $1982: 651$ ) La ponctuation serait ce qui reste du corps dans l'écrit. La ponctuation peut être liée à des émotions de personnages ou du sujet, mais elle est surtout la trace de cet « état d'arrièreplan du corps » que met à jour Damasio ${ }^{16}$, état d'arrière-plan qu'il oppose précisément à l'état émotionnel. Parmi les auteurs qui insistent sur cette notion de respiration et de corps, on trouve en particulier Louise Desbrusses, autrice et danseuse, qui souligne, dans 
une série d'essais sur les rapports de l'écrit et du corps, qu'on écrit forcément, toujours avec son corps ${ }^{17}$ :

L'expérience que je fais est celle d'écrire depuis mon corps, depuis la mémoire que ce corps a de telle sensation, perception, émotion, humeur ou disposition ou état d'esprit (ou de son absence, voire de sa privation) aussi infime soit-elles, que ce corps l'aie vécu directement ou par l'imagination. Pour transmettre par un texte la qualité propre à cette sensation, perception, émotion, humeur, disposition ou état d'esprit (ou de son absence, voire de sa privation) je dispose de mots et de signes de ponctuation (Desbrusses, $2015: 107$ ).

Les auteurs ou autrices qui écrivent pour le théâtre évoquent systématiquement ce rapport au corps, que ce soit Picault (citée en (17)), Efoui (qui évoque le "souffle») ou encore Siméon, selon lequel «la ponctuation est affaire de poumon et de mâchoire ». C'est le cas aussi de Bernard Noël, poète des Extraits du corps, qui écrit par ailleurs, dans Le Tu et le silence: « nous avons deux corps : l'un de chair, l'autre de mots; l'un qui s'exprime par le sexe, l'autre par la langue». Il s'agit bien, par la ponctuation, de trouver « une langue du corps » (Cottron d'Aubigné, qui associe ponctuation et «respiration, pause, énergie, rythme »). Qu'il s'agisse du corps qui écrit, du corps qui lit, ou du corps du texte, la ponctuation est perçue - en raison de son signifié rarement déterminé qui met par défaut l'accent sur le signifiant, en raison aussi de sa dimension iconique - comme laissant davantage place à la matérialité. S'opère ainsi par elle quelque chose de l'ordre d'une « intertextuation du corps » (Certeau, $1980: 219$ ), notion qui désigne le fait que « les êtres vivants sont "mis en texte", mués en signifiants ».

La réponse très précise de Siméon permet d'affiner cet enjeu. Après avoir précisé que ses réponses "varient franchement » selon le genre (essai d'un côté, poésie et théâtre de l'autre), les articles de réflexion relevant d'une "ponctuation traditionnelle», qui " organise et fixe la pensée », comme outil de mise au point, l'auteur indique qu'il en va tout autrement dans son travail de création :

(22) Il s'agit clairement - poésie ou théâtre - d'un autre système de ponctuation qui se construit et s'organise en se débarrassant de la ponctuation traditionnelle et de ses signes conventionnels car justement ils conceptualisent là où je veux du corps, du souffle, de l'incarnation. De l'oralité.

Donc j'utilise le vers «non ponctué »: ici tout est rythme, constitué par la variation de la longueur du vers, les coupes abruptes, les parataxes, les rejets et contre-rejets, enjambements etc. Et en effet le blanc est l'instrument le plus opérant de la ponctuation. Il s'agit bien de marquer "l'inquiétude du discours", donc l'inquiétude du sens qui est le propre de la poésie (le principe poétique étant la source sine qua non de mon écriture littéraire).

Il y a en outre une différence visible, et assumée, entre ma poésie (publiée à Cheyne) et mon théâtre (publié aux Solitaires Intempestifs). La raison en est probablement le degré d'oralité induit. Dans ma poésie, il y a oralité mais comme parole intérieure, voix du dedans qui a à voir avec le corps certes, chair et sang, mais dans la mi-voix; le corps comme origine (donc ses caractères y sont : souffle, « scansion du sang »...) signifie sa présence mais disons, discrètement.

Dans le théâtre, la langue prend en compte le corps en mouvement, la voix proférée: celle du comédien. Ces textes sont écrits pour le dire: d'où une ponctuation blanche très affirmée. Ponctuation du souffle sans conteste: arrêts, élans successifs, itérations, apnées, soudain déliements, stases, martèlements etc. [...] les comédiens me disent tous que c'est en mettant le texte en bouche que le sens apparaît. La ponctuation est donc affaire de poumon et de mâchoire! Ce qui à mes yeux vérifie l'efficacité de cette non-ponctuation ponctuante, c'est que dans les proférations de mes textes par des comédiens multiples, il y a très peu d'écarts dans leur lecture syntaxique : on entend bien le même texte. 
Dans une énumération significative, Siméon passe du corps au souffle à l'oralité. Il distingue l'oralité intérieure, endophasique, de la poésie, et l'oralité extérieure, proférable, au théâtre. C'est selon lui l'oralité intérieure, envisagée dès l'écriture, qui explique la part du blanc. Quand il n'y a pas de ponctuation noire ("vers non-ponctué", non-ponctuation ponctuante), « tout est rythme », grâce au blanc, indique l'auteur. Que la ponctuation soit envisagée comme silence ou effet de voix, voix du dedans ou favorisant une voix du dehors, elle a à voir avec l'effet de voix, la vocalité. La ponctuation noire serait davantage liée à l'effet de voix, la ponctuation blanche à la respiration: dans tous les cas, il y a rythme et la ponctuation est avant tout, pour les auteurs et autrices interrogé.e.s, cet espace, ce signe du rythme: organisation du mouvement de la parole par un sujet (Meschonnic), dans le continu du discours. Le mot rythme est ainsi utilisé par des auteurs aussi différents que Bégaudeau, Daeninckx, Deguy, Garron, Glykos, Kaplan, Macé, Massera, Picault, Samoyault et Ternaux.

En cas de blanchiment - suppression partielle ou totale des signes noirs attendus dans une syntaxe ou mise en place normée -, ou d'adoucissement - remplacement des ponctuations fortes par des ponctuation intermédiaires (virgules, points de suspension...) -, démarches fréquentes dans la littérature contemporaine et qu'on nomme parfois sousou dé-ponctuation, mots qui n'ont de sens que si on prend comme référence et horizon d'attente une ponctuation traditionnelle ${ }^{18}$, l'effet est celui d'une respiration accrue, d'une présence de la vocalité. Si l'on suit les analyses proposées par Philippe (2009), la dimension esthétique de l'oralité apparait dans la littérature avec Ramuz et Giono dans les années 1920-1930, Céline opérant un passage de l'oralité à la vocalité à partir des années 1930-1950. De nombreux critiques estiment que la littérature de la fin du XXe siècle met en avant la notion de "voix $»^{19}$. Or, depuis l'arrivée du magnétophone (popularisé dans les années 1950), la possibilité est donnée aux auteurs de penser la littérature hors du livre, soit pour oraliser un texte déjà écrit, soit pour enregistrer directement son texte sous la dictée, soit pour faire de la poésie sonore (qui nait dans les années 1950). À partir de Valéry et de Claudel, la présence de la voix, dans les œuvres comme dans les analyses, n'a cessé de s'affirmer au $\mathrm{XX}^{\mathrm{e}}$ siècle, ce que confirment les œuvres de Des Forêts, Beckett, Pinget, Barthes, Bonnefoy, les monologues de François Bon (Parking, Impatience) ou de Bernard Noël (La Comédie intime), etc., Michel Tournier affirme ainsi : "Quand on me parle de style à propos d'une œuvre littéraire, je pense d'abord à une voix, au timbre inimitable d'une voix $»^{20}$, et Marie Cardinal :

(23) les signes de la ponctuation sont là aussi pour indiquer des rythmes, des respirations, des tempo. Peut-être que dans l'écriture moderne la parole, la respiration, le silence, la durée, prennent une plus grande place que jadis ${ }^{21}$.

Il convient toutefois de ne pas confondre respiration et voix : «La voix, non la respiration, est la matière de l'oralité. La voix est ce qui disparait dans l'écrit, pas la respiration » (Meschonnic, 1982: 660). Il y aurait une respiration de l'écrit, que marquerait la ponctuation comme signe non verbal, et plus particulièrement le blanc typographique, comme signe non noir, se fondant dans le cadre qui l'accueille (la page, l'écran); or, de même qu' « un homme peut faire la grève de la faim, non de la respiration $»^{22}$, un texte peut faire la grève des mots, non de la ponctuation, la respiration ne se coupant pas :

La respiration ne se coupe pas, ou si elle est coupée c'est d'une façon qui ne manque pas d'engendrer quelque drame. Rien ne s'inscrit dans une coupure de la respiration, si ce n'est de façon exceptionnelle. La respiration se rythme, la respiration est pulsation, la respiration est alternance vitale, elle n'est rien qui 
permette sur le plan imaginaire de symboliser précisément ce dont il s'agit, à savoir

l'intervalle, la coupure ${ }^{23}$.

La respiration du texte est nécessaire, «alternance vitale». Pour un certain nombre d'auteurs, le mouvement du sens se formule ainsi du côté de la vitalité : "Organicité, vitalité » (Léon), « vie » (Rahmy) ; l'imaginaire physiologique de la ponctuation souligne bien à quel point celle-ci porte une expérience (d'écriture) qui est toujours aussi une expérience du corps, une expérience vitale.

\section{Conclusion}

Les réponses à cette enquête ne permettent pas d'identifier un changement massif d'imaginaire en 40 ans. L'imaginaire linguistique joue sur des durées longues, et l'historicité est sensiblement la même, sans changement majeur d'épistémè ; "La respiration, qui a aussi des variantes culturelles, selon les modes de vie, peut être admise comme un universel du corps. Elle n'est donc pas historique, mais biologique. On ne respirait sans doute pas très différemment au XVIII ${ }^{e}$ siècle en France, et au XVII ${ }^{e}$.» (Meschonnic, 1982: 660) ; ou plutôt si le changement est en cours, avec le développement du numérique et de l'écriture web, il n'a encore que minoritairement affecté les pratiques et encore moins les imaginaires. François Bon, considéré comme un des pionniers sur ce point, en raison de sa présence sur le web depuis 1997, indique quelques pistes des nouveaux enjeux de la ponctuation :

(24) 300 ans de typographie classique (mais c'est seulement mi ou fin XIX ${ }^{\mathrm{e}}$ que l'université commence à normaliser la ponctuation classique, le Rabelais que lisait Flaubert n'avait pas bougé depuis le $\mathrm{XVI}^{\mathrm{e}}$ siècle), c'est une histoire complexe et magnifique. Les usages de la césure (et ce que ça devient sur iPad ou pourquoi le Kindle dispose d'un algorithme de gestion des blancs qui lui évite la césure) ne s'en détachent pas. Une page belle est une sensation pratique et arbitraire. La ponctuation a appris à s'insérer là. Dans nos modes de lecture web, la même exigence rend quasi impalpable le travail de typo (sur ce site, Tiers Livre, les typos sont fournies par une couche payante, celle de l'entreprise TypeKit), de même que la vitesse d'affichage est aussi pour moi une politesse au lecteur, sur ordi comme sur tablette ou téléphone, et que donc, à l'instant où vous lisez cette page, vous êtes en fait sur le serveur américain CloudFlare. La politesse au lecteur, en écriture web, passe par d'autres critères complémentaires à ceux de la ponctuation.

Et même politesse, pour un blogueur, d'entrer son texte avec des apostrophes «typo » et des insécables. Spip le permet, mais Wordpress non. Il me semble qu'on a affaire à un déplacement global de paramètres, non pas qui relativise la place de la ponctuation, mais oblige à la considérer dans son contexte de publication. Ainsi, le rapport visuel à l'italique, aux guillemets, aux tirets, renforcera-t-il cette présence écran du texte, et nous apprenons à l'anticiper dès l'écriture, comme un élément organique de la ponctuation même.

François Bon évoque ici d'autres normes, techniques, «quasi impalpables » comme les blancs, les césures, les insécables programmés par les serveurs... La ponctuation est, écritil, "à considérer dans son contexte de publication ", comme une sorte de ponctuation éditorialisée ${ }^{24}$ en amont; la maitrise de ces outils techniques permet aux auteurs de prendre en compte ces potentialités ou contraintes dès l'écriture web, et d'anticiper sur cette "politesse au lecteur " que la ponctuation peut représenter. Cette enquête rend sensible qu'en ce début du XXI ${ }^{e}$ siècle l'imaginaire de la ponctuation relève encore d'un imaginaire de la langue (rapport au corps, à la voix, au rythme), signe que, dans le discours littéraire, une poétique de la ponctuation a pris le pas sur une logique de la 
ponctuation. Mais elle n'intègre pas encore la manière dont l'écriture web et la lecture sur écran vont, par une sorte de renouveau (contraint par les enjeux techniques) de l'attention porté aux matérialités, contribuer à la mise au jour d'autres signes (blancs, émoticônes...) et d'autres fonctions de la ponctuation, et à termes d'autres imaginaires. De même que cette enquête s'inscrivait dans la continuité de celle d'Annette Lorenceau, espérons qu'elle pourra servir de point d'appui à celles qui suivront.

\section{BIBLIOGRAPHIE}

ANIS, J., 1983, «Vilisibilité du texte poétique », Langue française, 59, Larousse, Paris, p. 88-102.

ANIS, J., CHISS, J.-L., PUECH, C., 1988, L'Ecriture : théories et descriptions, éditions Universitaires, Paris.

ANIS, J., 2004, « Les linguistes français et la ponctuation », L'Information Grammaticale, 102, p. 5-10.

ARABYAN, M. (dir.), 2016, L'Énonciation éditoriale, Semen, 41, Presses universitaires de FrancheComté, Besançon.

BIKIALO S., 2016, « Énonciation éditoriale et littérature exposée. Matérialités et dialogismes dans des œuvres affichées d'O. Rosenthal et de J.-C. Massera », Semen 41 (dir. M. Arabyan), p. 69-89.

BIKIALO, S., RAULT, J., 2016, « Ponctuation et rythme (graphique) » dans S. Pétillon et F. Rinck (éds), La Ponctuation à l'aube du XXI ème siècle, Lambert Lucas, Limoges.

BUTOR, M., 1972, « Propos sur l'écriture et la typographie », Communication et langages, 13, p. 18-19,

CANUT, C., 2007, « L'épilinguistique en question », dans G. Siouffi et A. Steuckardt (éds), Les Linguistes et la norme, Peter Lang, Berne, p. 49-72.

CATACH, N., 1980, « La ponctuation », Langue française, 45, Larousse, Paris, p. 16-27.

CATACH, N., 1985, «L'écriture et le signe plérémique », Modèles linguistiques, 7, p. 53-71.

CERTEAU, M. de, 1980, L'Invention du quotidien. I. Arts de faire, Gallimard, « folio essais », Paris.

DESSONS, G., MESCHONNIC, H., 1998, Traité du rythme des vers et des proses, Dunod, Paris.

DAMASIO, A. R., 1995, L'Erreur de Descartes, Odile Jacob, Paris.

DÜRRENMATT, J., 1998, Bien coupé mal cousu, PU Vincennes, Saint-Denis.

DÜRRENMATT, J., 2015, La Ponctuation en français, Ophrys, Gap-Paris.

DESBRUSSES, L., BIKIALO, S., 2015, « Dialogue autour de la ponctuation comme trace du corps qui écrit », Littératures, 72, PU Mirail, Toulouse, p. 105-118.

FAVRIAUD, M., 2000, La Ponctuation : la phrase - dans la poésie contemporaine (à partir des œuvres de du Bouchet, Jacottet, Stéfan), Thèse de doctorat, Université de Paris VIII.

FAVRIAUD, M., 2004, «Quelques éléments d'une théorie de la ponctuation blanche - par la poésie contemporaine », L'Information Grammaticale, 102, p. 18-23. 
FINTZ, C., 2009, « Les imaginaires du corps dans la relation littéraire », Littérature, 153, p. 114-131.

LEFEBVRE, J., 2007, La Note comme greffe typographique : étude linguistique et discursive, thèse de doctorat, Université Paris III.

LORENCEAU, A., 1980, « La ponctuation chez les écrivains d'aujourd'hui », Langue française, 45, La ponctuation, p. 88-97.

MESCHONNIC, H., 1982, Critique du rythme, Verdier, Lagrasse.

PÉTILLON, S., 2003, Les Détours de la langue : étude sur la parenthèse et le tiret double, Peeters, Leuvain.

PHILIPPE, G., 2002, Sujet, verbe, complément, Gallimard, Paris.

PHILIPPE, G., PIAT, J. (éds), (2009), La Langue littéraire. Une histoire de la prose en France de Gustave Flaubert à Claude Simon, Fayard, Paris.

RAULT, J., 2014a, «De la "pause” à la "valeur" en langue : grammaticalisation des signes de ponctuation, CMLF, en ligne sur http://www.linguistiquefrancaise.org/articles/shsconf/ abs/2014/05/shsconf_cmlf14_01076/shsconf_cmlf14_01076.html.

RAULT, J., 2014b, « La ponctuation : problématiques linguistiques », Le Français aujourd'hui, 187, p. 9-18.

RAULT, J., 2015, Poétique du point de suspension. Essai sur le signe du latent, éditions Cécile Defaut, Nantes.

SIMON, C., 1977, « Un homme traversé par le travail », La Nouvelle critique, 105, p. 32-46.

TESTENOIRE, P.-Y., 2016, « Sur la conceptualisation de la "langue écrite" dans les théorisations linguistiques du début du XX ${ }^{\mathrm{e}}$ siècle ", dans Dossiers d'HEL, SHESL, 9, Écriture(s) et représentations du langage et des langues, p. 34-46.

TOURNIER, C., 1977, «Essai de définition de la ponctuation et classement des signes », dans La Ponctuation. Recherches historiques et actuelles (dir. N. Catach et C. Tournier), C.N.R.S. - H.E.S.O., p. 223-243.

TOURNIER, C., 1980, « Histoire des idées sur la ponctuation, des débuts de l'imprimerie à nos jours ", Langue française, 45, Larousse, Paris, p. 28-40.

VÉDÉNINA, L.-G., 1989, Pertinence linguistique de la présentation typographique, Peeters / Selaf.

\section{NOTES}

1. Au sein de ce critère d'une littérature de création, plutôt en prose, la variété est grande (et volontaire), aussi bien en termes d'expérience et d'œuvre (entre des auteurs émergents avec un ou deux livres et des auteurs reconnus ayant plus d'une centaine d'œuvres publiées), de génération (de trente à quatre-vingt-dix ans), etc.

2. Catach (1985: 53-71). S'inspirant de Hjelmslev, elle distingue les "plérèmes ", éléments " pleins » de sens ou signes, se situant à la fois sur le «plan de l'expression » et sur le «plan du contenu », et « cénèmes », éléments « vides » de sens, se situant sur le seul « plan de l'expression. 3. Girard : «évidemment sonore »; Picault : « dimension sonore, voire musicale dans un premier temps »; Macé : «sonore/orale »; Emaz : «Une dimension sonore/orale, clairement »; Arlix et Kwahulé : « sonore»; Molia : «plutôt sonore»; Raharimanana : «Sonore avant tout et graphique également. Sonore qui n'est pas forcément orale, on peut écrire aussi le son.» 
4. Dans l'enquête de Lorenceau (1980: 89), 18 auteurs répondaient que la ponctuation avait plus de rapport avec l'oral qu'avec la syntaxe (14 avec la syntaxe et 7 répondaient les deux). Elle évoque toutefois un certain nombre d'auteurs sensibles au blanc, surtout dans la poésie mais aussi dans la prose.

5. Voir la synthèse de cette prise en compte de la dimension graphique dans Bikialo et Rault (2016).

6. Voir Butor (1972 : 18-19), et Cardinal : « le blanc est peu respecté par l'éditeur et l'imprimeur. Pour des raisons d'économie (principalement le prix du papier) ils ont tendance à tasser le texte. » (citée par Lorenceau, $1980: 92$ ).

7. G.-H. Aubertin, Grammaire moderne des écrivains français, 1861, Lacroix \& Verbeckhoven, Bruxelles, p. 8.

8. I. Serça, Esthétique de la ponctuation, Gallimard, 2012.

9. M. Foucault, L'Ordre du discours, Gallimard, 1971, p. 10.

10. Bordas dans son introduction au $n^{\circ} 16$ de Semen, « Le rythme de la prose » (2002:7), rappelle que Sauvanet, dans Le Rythme et la Raison I (2000), distingue une centaine de définitions du rythme.

11. Becker, Bénabou, Cottron d'Aubigné, Cusset, Dabitch, Ernaux, Girard, Glykos, Kwahulé, ou encore Noël.

12. Ainsi Ernaux: «souffle, respiration, pause»; ou Bénabou: «Respiration, énergie, pause, rythme ".

13. Rault (2014b) a toutefois nuancé cette imaginaire prosodique du mot pause en s'appuyant notamment sur Furetière et Beauzée.

14. Voir notamment les synthèses réalisées par Pétillon (2003) et Bikialo et Rault (2016).

15. Voir Serça, "La ponctuation est l'anatomie du langage". Maylis de Kerangal», Littératures $\mathrm{n}^{\circ} 72,2015$, p. 173-184.

16. A.R. Damasio, L'Erreur de Descartes (1995 : 208): « Un état d'arrière-plan [...] correspond à l'état du corps tel qu'il se présente entre des émotions. Lorsque nous ressentons de la joie, de la colère ou toute autre émotion, l'état d'arrière-plan a été remplacé par un état émotionnel. »

17. L. Desbrusses, «Du corps (\&) de l'écrit \#1», Inculte, $n^{\circ} 18,2009$ : «peut-on écrire sans son corps ? écrire contre son corps n'est pas encore écrire avec ? et dans ce cas, est-ce que toutes les écritures ne sont pas du corps?»; d'où la suggestion, l'alternative possible: «déplacer l'attention du c.o.r.p.s. (qu'on observe) au corps (qu'on est); du c.o.r.p.s. qu'on écrit au corps qui écrit ", p. 111-112.

18. Mauvignier explique qu'en raison de la sortie de son livre d'une seule phrase [Ce que j'appelle oubli, Minuit, 2011)] il doit répondre sans cesse à cette interrogation : pourquoi le livre n'a pas de ponctuation? À chaque fois, il répond que «non seulement il est ponctué, mais c'est un de ceux sur lequel cette question a été la plus importante ».

19. Martin, La Bande sonore, Corti, 1998 et Rabaté, Poétiques de la voix, Corti, 1999, en particulier.

20. Cité par Philippe, $2009: 84-85$

21. Marie Cardinal, citée par Lorenceau, 1980 : 92 . Certains critiques ont trop pris au premier degré (au sens physiologique et au niveau de l'auteur plus que du sujet de l'écriture) ce type d'idées, comme François Bernard Michel qui, dans Le Souffle Coupé - Respirer et Ecrire (Gallimard, 1984), évoque et perpétue les études sur l'influence de l'asthme sur l'œuvre de Marcel Proust, et cite Claude Roy, qui aurait expliqué la modification du rythme des vers de sa poésie lors d'un séjour en réanimation après une intervention pour un cancer bronchique. Il constatait que sa dyspnée entraînait inconsciemment l'écriture de vers plus rythmés et moins libres qu'avant, la recherche d'un rythme respiratoire régulier en somme.

22. Ricœur, Philosophie de la volonté. Le volontaire et l'involontaire, Aubier, 1949, p. 108. 
23. Lacan, séminaire du 20 mai 1959, cité par Meschonnic (1982 : 660-661) et Dürrenmatt (1998:

13).

24. Je pense ici à la notion d'« énonciation éditoriale » telle qu'elle a été repensée dans le numéro 41 (2016) de Semen dirigé par M. Arabyan.

\section{RÉSUMÉS}

Dans l'objectif d'actualiser une enquête sur la ponctuation effectuée par A. Lorenceau en 1980 auprès de quarante écrivains, l'article rend compte d'une enquête opérée auprès d'une quarantaine d'auteurs et d'autrices contemporains sur leurs visions et pratiques de la ponctuation. De la somme de ces discours épilinguistiques émerge ainsi un imaginaire contemporain de la ponctuation dans le discours littéraire en particulier dans le rapport à la dimension rythmique (sonore et visuelle) des ponctuations noire et blanche et aux normes scripturales.

With the aim of updating a survey conducted by punctuation A. Lorenceau in 1980 with forty writers, the article reports on an investigation carried out with forty authors and contemporary autrices their visions and practices of punctuation. The sum of these epilinguistic discourse emerges as a contemporary imagination of punctuation in literary discourse particularly in relation to the rhythmic dimension (audio and visual) of black and white dots and scriptural standards.

INDEX

Mots-clés : ponctuation à valeur expressive, ponctuation à valeur sémantique, littérature contemporaine

\section{AUTEUR}

\section{STÉPHANE BIKIALO}

Université de Poitiers - FORELL 